\title{
ETNOBOTÁNICA DE LA COMUNIDAD CAMPESINA "SANTA CATALINA DE CHONGOYAPE" EN EL BOSQUE SECO DEL ÁREA DE CONSERVACIÓN PRIVADA CHAPARRÍ - LAMBAYEQUE
} Tina Lerner Martínez ${ }^{1}$, Aldo Ceroni Stuva ${ }^{2}$ y Claudia E. González Romo ${ }^{3}$.

\begin{abstract}
Resumen
Resultan particularmente escasos los estudios etnobotánicos en los bosques secos del noroeste peruano como también los referidos a comunidades campesinas o mestizas sujetas a una mayor influencia modificadora del mercado. Asimismo, son pocos los ejemplos sobre comunidades campesinas que logran proteger sus terrenos y establecerlos como unidades de conservación reconocidas por el estado. En este sentido, como punto de partida para la formulación de proyectos de desarrollo dentro del Área de Conservación Privada Chaparrí, se llevó a cabo un estudio durante enero y setiembre del 2002, con la participación de los pobladores de la comunidad de Santa Catalina de Chongoyape, para identificar los recursos vegetales que se utilizan localmente. Se registraron 122 taxa de plantas útiles (103 géneros y 45 familias botánicas), además 8 géneros nuevos de plantas alimento de la "pava aliblanca" (Penelope albipennis). Por otro lado, se describen los vocablos y las expresiones locales referidas a las plantas. Además, Se recopiló información histórica y arqueológica para evidenciar que algunas plantas se siguen utilizando desde épocas precolombinas en el bosque seco del noroeste peruano.
\end{abstract}

Palabras claves: Bosque seco, Chaparrí, etnobotánica, Santa Catalina de Chongoyape

\begin{abstract}
There is a lack of ethnobotanic studies conducted in the dry forests of northern Perú and in peasant communities subjected to man"s major influencing action. Likewise, examples that show the influence of market economy on peasant communities are scarce, particularly those that show how a peasant community can establish a national conservation area in its territory as a means of protecting their land. In order to build the foundations of a future development project, an ethnobotany study, from January to September 2002 was conducted in the Chaparrí Private Area of Conservation which included the participation of the Santa Catalina de Chongoyape community. The goals were to identify local plants used by the population. 122 taxa of useful plants were registered (103 genera and 45 botanical families were identified). 8 new genera of plants used as food by the white-winged guan (Penelope albipennis) were found. A description of the local vocabulary and expressions was made as well as a compilation of historical and archaeological data records to establish the traditional uses of the plants of the zone.
\end{abstract}

Key words: Chaparrí, dry forest, ethnobotany, Santa Catalina de Chongoyape

\section{Introducción}

Los estudios de etnobotánica perse desarrollados en la zona norte del Perú son escasos (La Torre, 1998; Schjellerup et al., 2000; Ceroni, 2002; Fernández \& Rodríguez, 2003) y para los bosques secos semidensos y de sabana de Lambayeque no existen antecedentes. Por otro lado, uno de los aspectos más descuidados de la Etnobotánica es el estudio de los usos de las plantas por los campesinos o mestizos. A diferencia de las sociedades indígenas, que generalmente viven en núcleos geográfica y económicamente aislados de las sociedades nacionales, los grupos campesinos viven en comunidades conectadas con mercados regionales, nacionales e, incluso, internacionales; y representan la mayor parte de la población, de las así llamadas, culturas tradicionales (Toledo, 1993). Santa Catalina de Chongoyape es una comunidad campesina agrícola y ganadera, asentada en el bosque seco de Lambayeque a 60 kilómetros de la ciudad de Chiclayo, en un lugar clave de tránsito de costa a sierra y viceversa. Se diferencia por haber creado en sus territorios la primera Área de Conservación Privada en el Perú. El $86 \%$ del territorio comunal $(34,413$ hectáreas) ha sido asignado para su conservación desde diciembre de 1999. En ella se vienen realizando programas de reintroducción de animales endémicos en peligro de extinción, como es el caso de la "pava aliblanca" (Penelope albipennis Taczanowski). Dentro de sus objetivos, además de preservar la biodiversidad y prestar distintos "servicios ambientales" (mantenimiento del ciclo hidrológico, conservación de suelos, mitigación del calentamiento global) está el desarrollo de la población local contemplando una política de

\footnotetext{
${ }^{1}$ Herbario MOL. Universidad Nacional Agraria La Molina. Av. La Molina s/n Lima - Perú. Apartado postal 456. Lima 100. Correo electrónico: tinalerner@yahoo.com.

2 Departamento Académico de Biología Facultad de Ciencias. Universidad Nacional Agraria La Molina. Apartado 456. Lima 100. Correo electrónico: aceroni@lamolina.edu.pe

${ }^{3}$ Universidad Autónoma de Tamaulipas México. 13 Blvd. López Mateos 928, Cd. Victoria, Tamaulipas

México. Correo electrónico: cgonzale@uat.edu.mx
} 
aprovechamiento sostenible de su flora (Angulo, 2000). En este sentido, un estudio etnobotánico caracterizado por la participación comunal, que explora la diversidad vegetal y los conocimientos sobre las plantas, es necesario como punto de partida en el proceso de desarrollo. La investigación debe propiciar que los campesinos contemplen las alternativas para el aprovechamiento de su diversidad vegetal (recursos genéticos y bioquímicos) y las ventajas comparativas que representan la conservación de estos ecosistemas neotropicales. Además, es importante revalorar los conocimientos sobre el bosque seco que han persistido ante el proceso de aculturación, deforestación y avance de la frontera agrícola, y en cierta medida buscar su protección para el beneficio de la comunidad. Por lo expuesto, el presente estudio se refiere al registro de la utilización de los recursos vegetales por parte de la Comunidad Campesina de Santa Catalina de Chongoyape en el año 2002. Los datos están complementados con información histórica y cultural que brindan un contexto más amplio para el análisis sobre el conocimiento de las plantas y su estado de conservación, en el bosque seco del noroeste peruano. Los objetivos principales fueron: 1) identificar las plantas útiles de la zona y documentar sus usos; 2) aportar al conocimiento de la "etnoclasificación local"; y 3) documentar el uso tradicional de las plantas en el bosque seco del noroeste peruano.

\section{Materiales y métodos}

\section{Ubicación}

La Comunidad Campesina Santa Catalina de Chongoyape se localiza dentro del Área de Conservación Privada Chaparrí, entre los 6²8'39' -

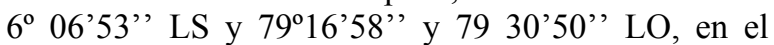
Distrito de Chongoyape, Provincia de Chiclayo, Departamento de Lambayeque (Figura 1).

\section{Descripción de la zona de estudio \\ Área de Conservación Privada Chaparrí}

Fue creada en diciembre del 2001 constituyéndose como la primera Área Natural Protegida de Conservación Privada del Perú con una extensión de 34,413 hectáreas y ocupando $86 \%$ del territorio de la comunidad. Inicia sus programas de conservación con el proyecto de reintroducción de la "pava aliblanca" Penelope albipennis a su ambiente natural (Angulo, 2002).

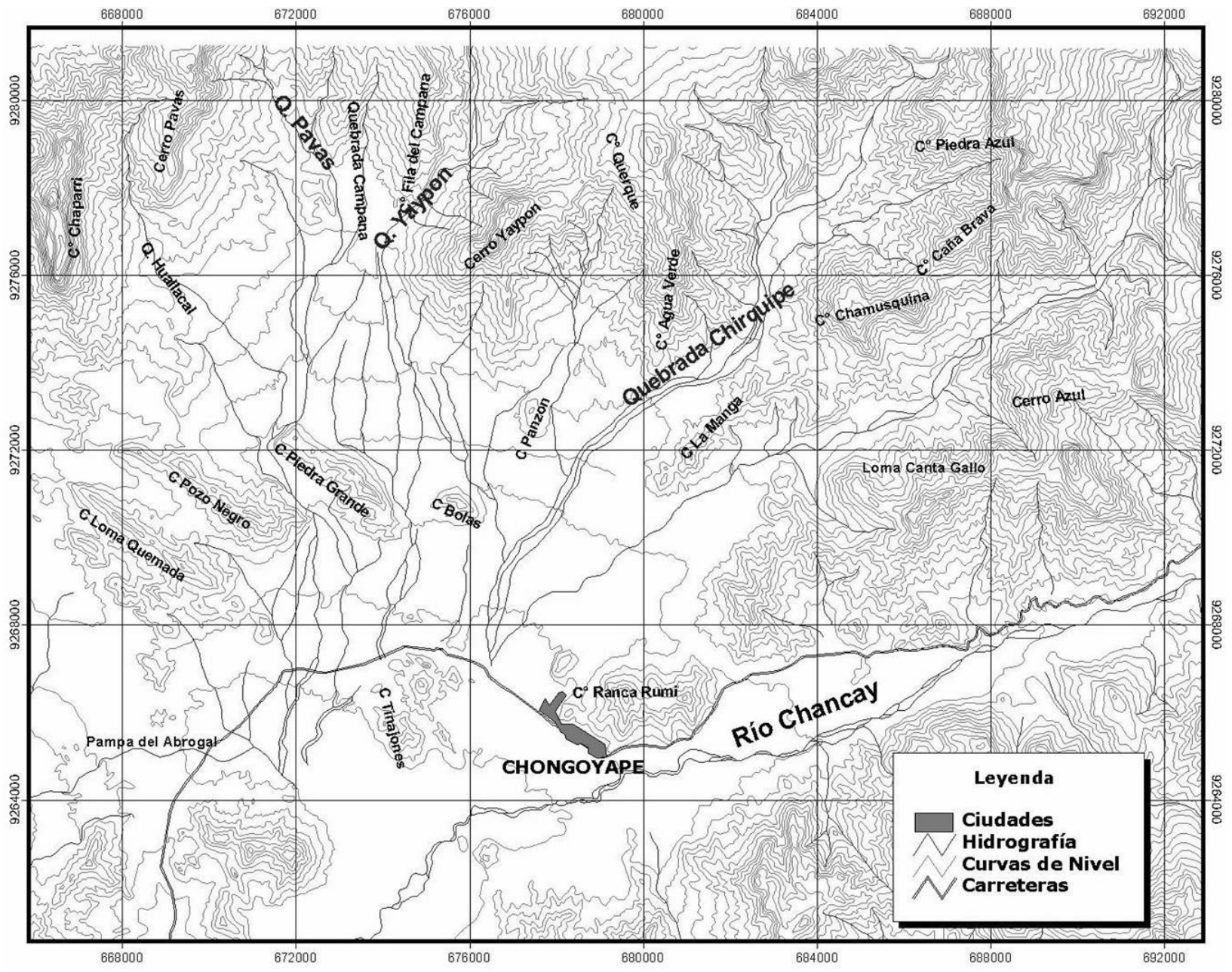

Figura 1. Mapa de ubicación de la zona de estudio. 


\section{Fisiografía e hidrología}

La zona comprende planicies desérticas (ca. $200 \mathrm{~m}$ de altitud) y bosque seco semi-denso (ca. $600 \mathrm{~m}$ ) en las laderas del contrafuerte andino occidental. Ocupa la cuenca media del Río Chancay-Lambayeque cuyas nacientes están en la laguna de Yanahuanca, en el departamento de Cajamarca.

\section{Clima}

Presenta un rango medio de temperatura entre 28.2 ${ }^{\circ} \mathrm{C}$ y $14.7{ }^{\circ} \mathrm{C}$, una precipitación media anual de 100 $\mathrm{mm}$ (registrándose más del $80 \%$ de este valor durante la estación húmeda). El fenómeno de "El Niño", asociado al aumento de la temperatura $\left(+2{ }^{\circ} \mathrm{C}\right)$ y precipitaciones (10 veces) en el departamento de Lambayeque, no ocurrió ese año (Angulo, 2002).

\section{Suelos}

La región posee tierras aptas para protección, que reúnen las condiciones mínimas requeridas para cultivo, pastoreo y producción forestal. Sin embargo, hay terrenos que permiten el uso continuo o temporal para sostener una ganadería económicamente productiva (ONREN, 1982).

\section{Ecología}

La zona de estudio corresponde a la ecorregión del bosque seco ecuatorial (Brack \& Mendiola, 2000) y presenta las siguientes zonas de vida: matorral desértico - Tropical, matorral desértico - Premontano Tropical, monte espinoso - Premontano Tropical (ONER, 1976). Además se consideran dos comunidades vegetales, bosque ralo semicaducifolio y bosque semidenso caducifolio (CDC, 1992).

\section{Metodología}

Se desarrollaron entrevistas semi-estructuradas contemplando dos grupos de entrevistados. Uno constituido por tres colaboradores locales claves (personas adultas con reconocida experiencia en el campo) para las caminatas etnobotánicas. Éstas se llevaron a cabo con cada colaborador en lugares representativos del bosque seco semi-denso caracterizados por una mayor diversidad florística y menor impacto antrópico. El segundo grupo, compuesto por 25 personas (niños, jóvenes y ancianos), para las entrevistas etnobotánicas en la zona de las viviendas y chacras. Se utilizaron listas de plantas de la zona, descripciones orales de las características más conspicuas de las mismas, fotografías y material botánico montado. Se tomó en cuenta para el registro de información el permiso de los colaboradores para colectar plantas, escribir, tomar fotografías y grabar 15 testimonios orales. El proyecto obtuvo la aprobación del presidente de la comunidad y se estableció un acuerdo de cooperación, verbal y por escrito, con colaboración de la gente local y el compromiso de retorno de los resultados obtenidos en el estudio a la comunidad. Para la documentación del uso tradicional de algunas plantas útiles del presente estudio se utilizaron registros arqueológicos de las culturas pre-colombinas que habitaban los bosques secos del noroeste peruano: material orgánico encontrado en tumbas (alimento, ofrendas funerarias u artefactos antiguos) y representaciones de plantas en cerámica (Yacovleff \& Herrera, 1934; Cabieses, 1974; Lumbreras, 1974; Hocquenghem, 1987; Polia, 1989; Ostolaza, 1998; Elera, 1990; Cárdenas et al., 1995; Correa, 2000). Además, los hallazgos científicos del Proyecto Arqueológico de Sicán (Universidad de Princeton New Yersey - Pontificia Universidad Católica del Perú - Museo Nacional Sicán, Lambayeque) en el valle del Río La Leche con respecto al uso de la vegetación combustible y la reconstrucción experimental de hornos alfareros u orfebres (Shimada \& Merkel, 1991; Shimada et al., 1994; Shimada \& Wagner, 2001). Se consideró además material histórico recopilado a partir de la conquista española (Valdizán \& Maldonado, 1923; Mateos, 1954; Kauffman, 1973; Rostworowski, 1981,1988).

\section{Resultados y discusión}

\section{Registro Etnobotánico}

En este estudio se reporta 122 taxa de plantas útiles (103 géneros y 45 familias botánicas) de las cuales una es un helecho y el resto angiospermas. La mayor cantidad de especies fueron silvestres principalmente distribuidas en el bosque ribereño restringido a las faldas de los cerros. En segundo lugar, las domesticadas en los llanos cercanos a los caseríos y luego las malezas que crecen en hábitats perturbados sin depender de los humanos para su sobrevivencia. En menor proporción se encontraron plantas asilvestradas y semicultivadas (Figura 2). Las familias botánicas más representativas en cuanto a número de especies útiles fueron Fabaceae con 17,2\%, Poaceae con 9\%, Asteraceae, con 6,5\%, Cactaceae con $5,7 \%$, Solanaceae con $5,7 \%$, Convolvulaceae con $3,3 \%$ y Anacardiaceae con $2,5 \%$ (Tabla 1). Las tres familias mejores representadas son las que presentan mayor número de géneros y especies neotropicales (Gentry, 1993; Maas et al., 1998; La Torre, 1997; Schjellerup et al., 2000).

Asimismo, se registraron 10 categorías de uso: alimenticia con 38 especies útiles, medicinal con 37 , combustible con 16 , construcción de viviendas, cercos y corrales con 21, tecnología local con 22, comerciales con 16 y agroforestería con 9. También se reconocieron 8 especies melíferas, 40 que sirven de forraje a los animales domésticos y 21 como alimento de la "pava aliblanca" (Penelope albipennis) (Figura 3). La mayor proporción de especies comestibles y medicinales refleja los satisfactores básicos de la población (Hernández et al., 1991; Schjellerup et al., 2000; Ceroni, 2002), y las especies forrajeras, el potencial ganadero de la zona (ONERN, 1982). 


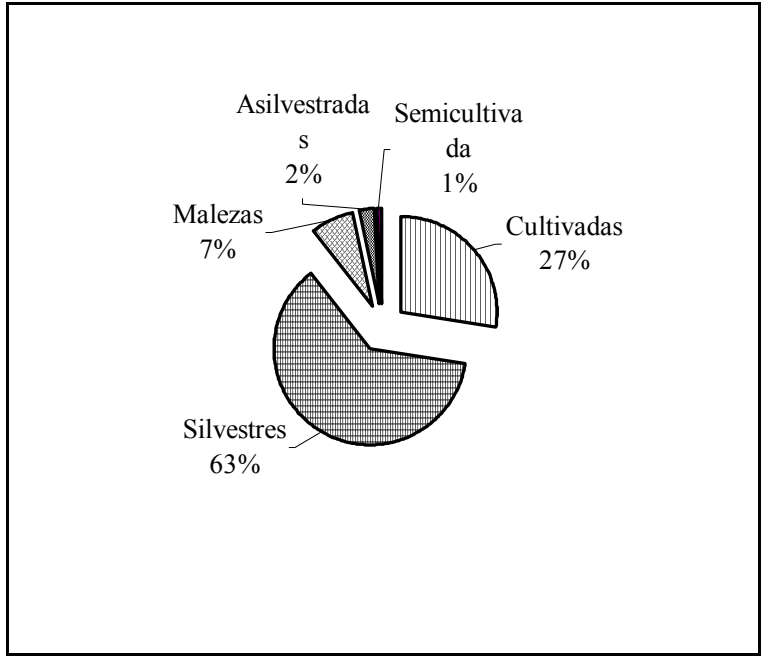

Figura 2. Porcentajes de especies cultivadas y asilvestradas en Santa Catalina de Chongoyape.

En cuanto al alimento de la "pava aliblanca" ( $P$. albipennis) en el Área de Conservación Privada Chaparrí se reportan 8 nuevos géneros de plantas: Bastardia, Corchorus, Loxopterygium, Melocactus, Momordica, Prunus, Solanum y Verbesina, (Ortiz, 1980; Ortiz \& Purisaca, 1981; Ortiz \& Díaz, 1997; Zevallos \& Ríos, 1998). Se tomó en cuenta a los individuos en estado de semicautiverio o en proceso de reintroducción que son monitoreados periódicamente en el área de conservación. Estos crácidos están iniciándose en el forrajeo de plantas silvestres, principalmente las de quebrada (como son los árboles Pithecellobium excelsum (Kunth) C.Martius y Muntingia calabura L., y las arbustivas Acnistus arborescens (L.) Schlecht. y Carica parviflora (A. DC.) Solms, entre otras); por otro lado dos especies cultivadas (Coffea arabica L. y Prunus avium L.) forman parte de la alimentación en semicautiverio.

Si bien existen conocimientos sobre el uso de los vegetales, en muchos casos éstos no tienen un carácter práctico. Algunos testimonios locales refieren la pérdida de la utilización del vegetal, que observaron o aprendieron de las generaciones pasadas, por la escasez del recurso, un mal manejo o su reemplazo por productos sintéticos, entre otras causas.

El uso de las plantas y la aplicación del conocimiento están cada vez más limitados por la lejanía y escasez del recurso vegetal. En efecto, el bosque seco de Lambayeque está en proceso de desertificación dada la ampliación de la frontera agrícola y tala indiscriminada (Proyecto Algarrobo, 1997).

En el caso de las plantas medicinales, existe una tendencia a reemplazar las prácticas etnomedicinales por la utilización de medicamentos sintéticos. Sin embargo, cuando la medicina moderna falla y la enfermedad es grave, los pobladores acuden donde los "curanderos" campesinos que preservan una gran farmacopea basada en plantas nativas (Prance, 1991).

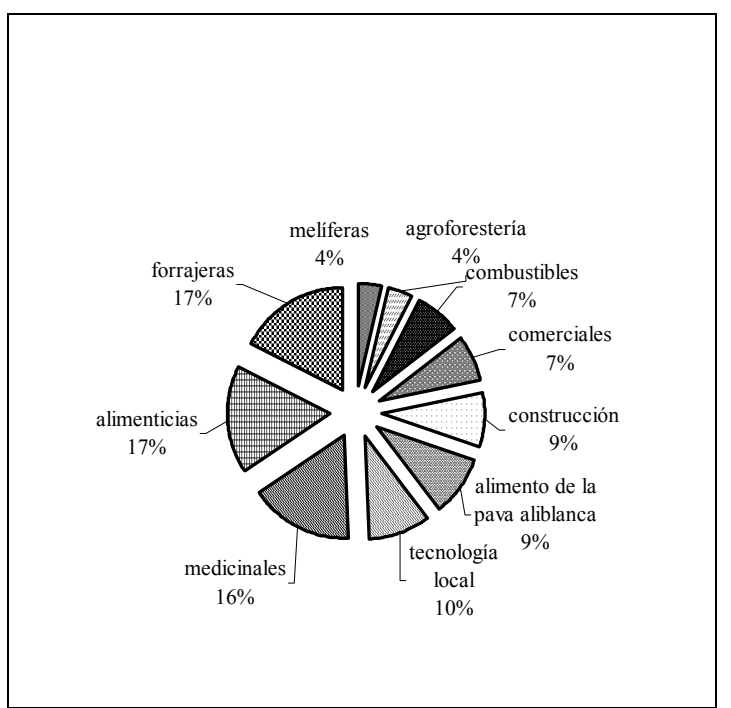

Figura 3. Porcentajes de especies vegetales según la categoría de uso

\begin{tabular}{|} 
Tabla 1. Número de especies de plantas útiles por familia \\
\hline $\mathbf{N}^{\mathbf{0}}$ & Familia & $\mathbf{N}^{\mathbf{0}}$ de especies \\
\hline 1 & Acanthaceae & 1 \\
\hline 2 & Amaranthaceae & 1 \\
\hline 3 & Anacardiaceae & 2 \\
\hline 4 & Annonaceae & 3 \\
\hline 5 & Apocynaceae & 1 \\
\hline 6 & Arecaceae & 8 \\
\hline 7 & Asteraceae & 1 \\
\hline 8 & Asclepiadaceae & 1 \\
\hline 9 & Bixaceae & 2 \\
\hline 10 & Bombacaceae & 1 \\
\hline 11 & Boraginaceae & 7 \\
\hline 12 & Burseraceae & 3 \\
\hline 13 & Cactaceae & 2 \\
\hline 14 & Capparaceae & 5 \\
\hline 15 & Caricaceae & 3 \\
\hline 16 & Convolvulaceae & 1 \\
\hline 17 & Cucurbitaceae & 21 \\
\hline 18 & Cyperaceae & 1 \\
\hline 19 & Elaeocarpaceae & 2 \\
\hline 20 & Euphorbiaceae & 1 \\
\hline 21 & Fabaceae & Lamiaceae & 1 \\
\hline 22 & Lauraceae & Liliaceae & 1 \\
\hline 23 & Loranthaceae & 1 \\
\hline 24 & & \\
\hline 25 & & 1 \\
\hline
\end{tabular}




\begin{tabular}{|c|c|c|}
\hline 26 & Malvaceae & 2 \\
\hline 27 & Moraceae & 1 \\
\hline 28 & Musaceae & 2 \\
\hline 29 & Myrtaceae & 2 \\
\hline 30 & Nyctaginaceae & 3 \\
\hline 31 & Passifloraceae & 3 \\
\hline 32 & Piperaceae & 2 \\
\hline 33 & Poaceae & 11 \\
\hline 34 & Pteridophyta & 1 \\
\hline 35 & Rosaceae & 2 \\
\hline 36 & Rubiaceae & 1 \\
\hline 37 & Rutaceae & 2 \\
\hline 38 & Sapindaceae & 1 \\
\hline 39 & Solanaceae & 7 \\
\hline 40 & Scrophulariaceae & 3 \\
\hline 41 & Sterculiaceae & 1 \\
\hline 42 & Tiliaceae & 1 \\
\hline 43 & Ulmaceae & 1 \\
\hline 44 & Verbenaceae & 2 \\
\hline 45 & Vitaceae & 1 \\
\hline
\end{tabular}

\section{Etnoclasificación local}

Se registraron 24 vocablos del habla local en 7 categorías etnobotánicas (Lerner, 2003). Se encontraron voces de origen español y quechua que denotan el carácter mestizo de los pobladores de la comunidad de Chongoyape dada la migración desde la sierra de Cajamarca (Tocmoche y Santa Cruz) a Chongoyape. De origen español tenemos "tabardillo" que significa insolación; americanismos como "chacla" que significa en el Perú, caña muy fuerte; del quechua "quincha", kincha, que significa pared de ramas o cañas; empalizada; pared; cerca; muro; corral; y "siso", sisa que significa polen, entre otras. Además, el léxico está influenciado por el entorno predominantemente agrícola y ganadero del valle costero y muchos de los nombres asignados localmente a las plantas, son de carácter descriptivo y resultan de una conjunción de información diversa (formas, hábitos, usos, etc.) sobre cada especie.

\section{Documentación del uso tradicional de las plantas en el bosque seco del noroeste peruano}

Las fuentes arqueológicas e históricas utilizadas logran establecer parcialmente enlaces tradicionales en el uso de las plantas del bosque seco dado que no es posible la verificación botánica de la especie. En este sentido, se sugiere que, desde tiempos precolombinos hubo continuidad en la utilización de Prosopis pallida (H \& B ex Willd.) H.B.K. como combustible y en la construcción, como también en las prácticas curanderas el uso de Echinopsis peruviana (Britton \& Rose) Friedrich \& G.D. Rowley desde el periodo
Cupisnique (1500-100 a.e.c) hasta la Colonia (15351824). Asimismo, el cultivo y la alimentación con Zea mays L. desde el periodo Moche (0-800 e.c) hasta la Colonia.

Actualmente, encontramos que la especie preferida como leña para cocinar y como postes en la construcción es Prosopis pallida (H \& B ex Willd.) H.B.K. "algarrobo", además de Loxopterigium huasango Spruce ex Engles "hualtaco", debido a su gran rendimiento como combustible y alta durabilidad como elemento estructural de la vivienda. Asimismo, Zea mays L. "maíz" constituye la base alimentaria, junto a Oryza sativa L. "arroz", plantas a las cuales el campesino le destina la mayor fuerza de trabajo por su valor comercial. Por otro lado, el cactus alucinógeno Echinopsis peruviana (Britton \& Rose) Friedrich \& G.D. Rowley "San Pedro" es utilizado en la zona por seis curanderos, quienes guardan la sabiduría acerca del proceso de colecta y preparación de la planta y la sanación de los pacientes enfermos. Sin embargo, la dimensión sacramental y la modalidad de su uso han cambiado según las tendencias y exigencias del medio; actualmente, el aspecto económico y monetario juega un papel determinante en las sesiones de curación.

Por otro lado, el manejo sostenible de las plantas combustibles ha ocurrido desde el periodo Sicán (9001100 e.c) hasta el Chimú (1100 e.c- 1480) dada la producción alfarera y orfebre a gran escala. Los antiguos consideraban una mayor diversidad de especies combustibles (arbóreas, arbustivas y herbáceas), una forma racional de cosecha de la leña (principalmente ramas) y la eficiencia de los hornos. También, la abundancia de los árboles, sobre todo del "algarrobo" por su alto poder calorífico, fue probablemente el factor clave para la presencia de centros cerámicos y metalúrgicos (Shimada \& Wagner, 2001). Además, se debe considerar que el manejo racional fue posible dada la organización social-política-religiosa pre-colombina y la menor densidad poblacional con respecto a nuestra era.

\section{Conclusiones}

1. En el Área de Conservación Privada Chaparrí se encontraron 124 taxa de plantas útiles pertenecientes a 104 géneros y 45 familias botánicas, todas angiospermas y una sola pteridofita. $\mathrm{Y}$ en cuanto a las familias botánicas, Fabaceae y Poaceae registraron mayor número de especies útiles.

2. Se reportan 40 taxa de plantas forrajeras, 38 alimenticias, 37 medicinales, 22 utilizadas en la tecnología local, 21 alimento de la "pava aliblanca" (Penelope albipennis), 20 usadas en la construcción, 16 combustibles, 9 agroforestales y 8 melíferas, lo que permite identificar qué especies son importantes para la gente de Santa Catalina de Chongoyape para su manejo, conservación y administración dentro del Área Natural Protegida de la comunidad. 
3. Las categorías de uso mejor representadas con especies vegetales fueron las forrajeras, alimenticias y medicinales. Esto se relaciona con las principales actividades económicas del poblador, la agricultura y la ganadería, sin embargo las prácticas etnomedicinales, entre otros conocimientos, están siendo afectadas por la ampliación de la frontera agrícola y los recursos silvestres están cada vez más lejanos y escasos.

4. Bastardia, Corchorus, Loxopterygium, Melocactus, Momordica, Prunus, Solanum y Verbesina, son los 8 nuevos géneros de plantas que se reportan como alimento de la "pava aliblanca" (Penelope albipennis) encontrados en la zona de semicautiverio y reintroducción de esta ave en el Área de Conservación Privada Chaparrí.

5. Los 24 vocablos del habla local reportados en 7 categorías etnobotánicas son voces de origen español y quechua, denotando el carácter mestizo de los pobladores de la comunidad de Santa Catalina de Chongoyape.

6. Las fuentes arqueológicas e históricas utilizadas logran establecer parcialmente enlaces tradicionales en el uso de las plantas del bosque seco dado que no es posible la verificación botánica de la especie. En este sentido, se sugiere principalmente, desde tiempos precolombinos, la continuidad en la utilización de Prosopis pallida como combustible y en la construcción, de Zea mays en la alimentación y cultivo, y de Echinopsis peruviana en las prácticas curanderas.

\section{Agradecimientos}

Los autores expresan su agradecimiento a todos los amigos de Santa Catalina de Chongoyape que colaboraron en el estudio de campo, especialmente a Pedro Cáceres, José Cruz, Felipe Diaz e Iván Vallejos. A la Asociación Cracidae Perú por el apoyo financiero, especialmente a Fernando Angulo por la logística. A los Herbarios de la Universidad Nacional Agraria La Molina (MOL), Universidad Nacional Mayor de San Marcos (USM), Universidad Privada Antenor Orrego y Universidad Nacional de Trujillo por su acogida. A nuestro amigo David Goldstein por su hospitalidad en el valle de La Leche y apoyo con la información del Proyecto Arqueológico de Sicán.

\section{Literatura citada}

Angulo F. (editor). 2002. Área de Conservación Privada Chaparrí. Plan Maestro.

Brack A. \& Mendiola C. 2000. Ecología del Perú. PNUD. Ed. Bruño. Lima, Perú.

Cabieses F. 1974. Dioses y enfermedades: la medicina en el antiguo Perú. Tomo I. Ed. Arte gráfico. Lima.

Cárdenas J., Rodríguez J. \& Aguirre L. 1995. El material orgánico en Huaca de la Luna. Páginas 129-149. en: Investigaciones en la Huaca de la
Luna. Ed. Facultad de Ciencias Sociales de la Universidad de la Libertad, Trujillo. Perú.

Ceroni A. 2002. Datos etnobotánicos del poblado de Huaylingas, cuenca la Gallega, Morropón, Piura. Revista Ecología Aplicada Departamento Académico de Biología.UNALM. 1(1): 65-70.

CENTRO DE DATOS PARA LA CONSERVACIÓN (CDC). 1992. Estudio de Conservación de la Diversidad Natural de la Región Noroeste del Perú. UNALM.

Correa R. 2000. Arquitectura rural en la costa norte Túcume: continuidad y mestizaje. Revista de Turismo y patrimonio. Universidad San Martín de Porres. Lima. (1)1: 69-81.

Elera C. 1990. El complejo cultural cupisnique: antecedentes y desarrollo de su ideología. Páginas 229-257. en: Senri Ethnological studies No37 'El mundo ceremonial andino'. National Museum of Ethnology. Japón.

Fernández A. \& Rodríguez E. 2003. Etnobotánica del Perú pre-hispano. Public. Del Orthus. Bot. Truxillense (HBT) UNT. Perú (en prensa).

Gentry A.H. 1993. A field guide to the families of woody plants of Northwest South America (Colombia, Ecuador, Peru) with supplementary notes of herbaceous taxa. Washington, D.C.: Conservation International.

Hernández L., González C. \& González F. 1991. Plantas útiles de Tamaulipas, México. Anales del Instituto de Biología de la UNAM. Ser. Bot. 62(1): $1-38$.

Hocquenghem A. 1987. Iconografías mochica. PUCP Fondo editorial. Lima.

Kauffman D.F. 1973. Manual de Arqueología peruana. Ed. Peisa. Lima.

La Torre M. 1997. Etnobotánica de los Recursos Vegetales Silvestres del Caserío de Yana cancha, Distrito de Cumucho, Provincia de Celendín, Departamento de Cajamarca. Tesis para optar al título de Bióloga. UNALM.

Lerner T. 2003. Etnobotánica de los Recursos Vegetales de la Comunidad 'Santa Catalina de Chongoyape', Microcuenca del Río Chanchay, Distrito de Chongoyape, Provincia de Chiclayo, Departamento de Lambayeque. Tesis para optar el grado de Biólogo. Universidad Nacional Agraria La Molina.

Lumbreras L.G. 1974. Los orígenes de la civilización en el Perú. Ed. Milla Batres. Lima-Perú.

Maas J.M., Westra L.Y.Th. \& Farjon A. 1998. Familias de Plantas Neotropicales, una guía concisa de las familias de plantas vasculares neotropicales. (Germany): Koeltz Scientific Books.

Mateos P.F. (Editor). 1954. Historia del nuevo mundo. Obras del Padre Bernabé Cobo. Compañía de Jesús. Madrid. 
OFICINA NACIONAL DE EVALUACIÓN DE RECURSOS NATURALES (ONERN). 1976. Mapa Ecológico del Perú. Guía explicativa. Lima. Mapa escala 1:1'000,000.

1982. Clasificación de las tierras del Perú. Lima. Mapa a escala 1: 1'000,000.

Ortiz E. 1980. Estudio preliminar sobre la pava aliblanca (Penelope albipennis). Lima. No publicado.

\& Purisaca J. 1981. Estudio preliminar sobre

la Pava aliblanca (Penelope albipennis) Taczanowski 1877. Primer Simposio de la familia Cracidae. UNAM. México. \& Diaz V.R. 1997. Estudio de Campo y Reevaluación de la Población de Pava Aliblanca (Penelope albipennis). Pp. 218-232 En: The Cracidae: their Biology and Conservation (S.D. Strahl, S. Beaujon, D.M. Brooks, A.J. Begazo, G. Sedaghatkish and F. Olmos). Hancock House Publ.

Ostolaza C. 1998. Etnobotánica V: La cultura Moche. Quepo, Sociedad Peruana de Cactus y Suculentas. (Lima). 12: 62-68.

Polia M. 1989. Las lagunas de los encantos: Medicina tradicional andina del Perú septentrional. CEPESER. Piura, Perú.

Prance G. 1991. What is Ethnobotany today? Journal of Ethnopharmacology. 32: 206-209.

PROYECTO ALGARROBO. 1997. Políticas para la gestión sostenible de los bosques secos de la costa norte del Perú (en prensa).

Schjellerup I., Espinoza C., Quipuscoa V. \& Fjeldsa J. (editores). 2000. Yanchalá - La gente y la biodiversidad. DIVA, Technical Report 10. Ecología y Desarrollo 9.

Shimada I. \& Merkel J.F. 1991 Copper Alloy Metallurgy in Ancient Perú. Scientific American. 265(1): 80-6
Elera C., Chang V., Neff H., Glascock M., Wagner U. \& Gebhard R. 1994. Hornos y producción de cerámica durante el periodo formativo en Batán Grande, costa norte del Perú. Páginas 67-372. en: Tecnología y organización de la producción de cerámica prehispánica en los Andes. PUCP. Lima.

\& Wagner U. 2001. Peruvian Black Pottery Production and Metalworking: A Middle Sicán Craft Workshop at Huaca Sialupe. MASCA Journal. 26(1): 25-30.

Rostworowski De Diez Canseco M. 1981. Recursos Naturales Renovables y Pesca Siglos XVI y XVII. Instituto de Estudios Peruanos, Lima.

1988. Historia del Tahuantinsuyu. Instituto de Estudios Peruanos. Lima.

Toledo V. 1993. La Racionalidad Ecológica de la Producción Campesina. Páginas 197-218. en: Ecología, Campesinado e Historia. Sevilla, E. y González de Molina, M. (editores). La Piqueta, Madrid.

Valdizán H. \& Maldonado A. 1923. La medicina popular peruana. Tomo II. Editorial Torres Aguirre. Lima.

Yacovleff E. \& Herrera F. 1934. Revista del Museo Nacional (Lima). 3: 241-323.

Zevallos P.A. \& Ríos J. 1998. Determinación botánica de 24 especies arbóreas del Departamento de Lambayeque. Páginas 319-342. en: Bosques secos y desertificación. Memorias del seminario internacional. Proyecto Algarrobo - INRENA. Lambayeque, Perú. 